\title{
Dendrimers: a Versatile Smart Nanoplatform for Advanced Nanotechnology Applications
}

\author{
Domenico Lombardo \\ CNR-IPCF, Consiglio Nazionale delle Ricerche, Istituto per i Processi Chimico-Fisici, \\ Viale Ferdinando Stagno d' Alcontres, Messina, Italy \\ lombardo@ipcf.cnr.it
}

\section{Extended Abstract}

Dendrimers are highly branched artificial (macro)molecules obtained by stepwise controlled, reaction sequences which are characterized by a combination of a high number of functional groups and a compact molecular structure [1]. The ability to be engineered for specific applications, through the choice of the interior (and core) molecular region, and peripheral surface, makes dendrimers versatile tools for the investigation of the molecular organisation, at the nano-scale level, during the bottom-up synthesis of functional nanostructures. The dendrimer structural features possess improved quality of physical, chemical, and biological properties such as enhanced colloidal stability, solubility, ability to work as compound delivery systems, and many others [1-3]. More specifically, dendrimers have gained a broad range of applications in supramolecular chemistry, particularly in host-guest reactions and self-assembly processes, while applications highlighted in recent investigations include nanocarriers drug delivery, gene transfection, catalysis, energy harvesting, rheology modification, and nanoscale science and technology [1-4].

The formation of nanoparticles systems with well-defined sizes and shapes creates room for possible control over architectural design (branching, density and surface functionality) and is of prominent interest for advanced biomedical applications [5-10]. More specifically, the possibility of encapsulating drug molecules within the branches of dendrimers offers the potential of dendrimers to interact with poorly soluble drugs, enhance drug stability, bioavailability and control of drug release. For those reasons, dendrimers have become an ideal delivery vehicle candidate for the investigation of the effects of polymer size, charge, and chemical composition on biological environments, such as lipid bilayer interactions [610], internalization and biodistribution, blood plasma retention time [5]. Moreover, their ease of preparation and functionalization allow the possibility to attach multiple surface groups that renders dendrimers as suitable vehicles for theranostic biomedical applications [3, 4].

Besides biomedical applications dendrimers can be used to improve many industrial processes. The combination of high surface area and high solubility makes dendrimers useful as nanoscale catalysts, In this respect, their many active sites (exposed towards the reaction mixture) enhance the possibility of encapsulating a single catalyst site whose activity can be enhanced by the dendritic superstructure [11]. Dendrimers has gained some attention also in environment friendly industrial processes, as they can encapsulate insoluble materials, such as metals, and transport them into a solvent within their interior, while the dendrimer supported surfaces could act as useful adsorbents for (electro-)analytical procedures in environmental studies [11]. Moreover, linear or branched dendrimer compounds have been used for the incorporation of specific components and serve as hole-injecting, hole-transporting, electron-transporting, or hole-blocking materials for organic electronic devices, such as organic thin-film transistors, organic solar cells and organic optical detectors [11, 12]. Recently, various dendrimenrs-based nanostructures are used in the development of (inexpensive) miniaturized, ultrasensitive and rapid environmental monitoring devices, and in the sensing of chemical and biological species [11, 12].

In table 1, are reported some of the main dendrimers promising applications in the field nanotechnology.

In conclusion, Despite the interesting results obtained in the last decades, dendrimers is continuing to stimulate a large variety of renewed interdisciplinary nanotechnology investigations, mainly focussed in may biomedical applications (including drug delivery, detection, diagnosis, and treatment of cancer, viral, and bacterial diseases) as well as industrial processes, including catalysis, micro fabrication, optical data storage, environmental remediation, adsorption, printing/photography and membrane filtration [13-15]. 
Table 1: Main Advanced Nanotechnology Applications of Dendrimers: Biomedical and Health, Electronics, Engineering, Consumer Goods and Environmental Applications.

\section{Biomedical and Healthcare Applications \\ - Diagnostics and Analysis \\ - Cellular Transport \\ - Targeted delivery (e.g. protein, antibody and anti-inflammatory; nanoparticles, radionucleides, fluorescent markers, etc.) \\ - MRI contrast agents (e.g. organ, vascular and tumour imaging) \\ - Transfection reagents, DNA-carriers \\ - Protein / enzyme mimics or modelling \\ - Development of topical microbicide creams; antimicrobial, \\ antiviral, antiparasitic agents \\ - polyvalent dendrimer-based drugs \\ - Artificial antibodies and biomolecular binding agents,}

\section{Engineering Applications}

- Chemical / biological sensors \& detectors

- Carbon fibre coatings and ultra thin films

- Polymer and plastics additives (e.g. for lowering viscosity, increasing stiffness, incorporating dyes, compatibilisers, etc.)

- Building blocks for nanostructured materials,

\section{Electronics Applications}

Molecular electronics for data storage

- 3-D optical materials

- Light-harvesting systems

- OLEDs (i.e. flat panel displays and other light

emission applications)

- Quantum dots

- Liquid crystals

- Printed wire boards

- Low-k materials (i.e. insulation materials)

\section{Consumer Goods Applications}

- Ink / laser-printing toners

- Dyes and paints, and Industrial adhesives

- Manufacture of nanoscale batteries and

lubricants

\section{Environmental Applications}

- Decontamination agents (trap metal ions)

- Ultrafiltration

In conclusion, the wide range of controllable dendrimers properties (such as size, shape, surface chemistry, architecture and chemical composition) provide an excellent tool for the design and engineering of macromolecular and supramolucular nano-structures for the development of advanced nanodevices in the field bio-nanotechnology. However, the optimization of the products and benefits (and the reduction of risks) request a long-term investment toward a wide range of nanotechnology applications. In recent years, in the field of biomedical applications, several dendrimers nano-platforms have led to many active preclinical investigations in the areas of inflammation, infectious diseases, oncology and ocular disease/wound healing [16, 17]. Despite the novel dendrimer-based products will have to face lengthy clinical trials before to be inserted in the market, we expect that in the next future a number of the current potential applications would probably be either in the applied R\&D phase or approaching the first commercial applications. For many applications (e.g., electronics, optical, medical), it is key to work with high purity products, with highly reproducible properties. Moreover, in upcoming dendrimer-nanotechnology applications, the price is unlikely to be a major barrier. In this respect a substantial efforts should be put for the research and development of novel synthesis methods (with reduction of steps or combined convergent/divergent approaches), that may reduce their price and ameliorate the level of the purity of the products ready for the market. Finally, the identification of the fundamental factors involved in the hierarchical self-assembly and supramolecular chemistry processes of dendrimers-nanostructures represents the initial step that drives towards the development of novel protocols for the construction of advanced functional materials.

\section{References}

[1] Dendrimers and Other Dendritic Polymers, Jean M.J. Frechet and Donald A. Tomalia, J. Wiley \& Sons (2001)

[2] D. Lombardo, "Modeling Dendrimers Charge Interaction in Solution: Relevance in Biosystems," Biochem. Res. Int. vol. 2014, 837651, 2014,

[3] D. Lombardo, P. Calandra, L. Pasqua, S. Magazù, "Self-Assembly of Organic Nanomaterials and Biomaterials: The Bottom-Up Approach for Functional Nanostructures Formation and Advanced Applications," Materials, vol. 13, pp. 1048, 2020.

[4] J.M.J. Fréchet, "Dendrimers and other dendritic macromolecules: From building blocks to functional assemblies in nanoscience and nanotechnology," J. Polym. Sci. vol. 41, pp. 3713-3725, 2003. 
[5] A.S. Abd-El-Aziz, C. Agatemor, "Emerging Opportunities in the Biomedical Applications of Dendrimers," J Inorg Organomet Polym, vol. 28, pp. 369-382, 2018.

[6] D. Lombardo, "Liquid-like ordering of negatively charged poly (amidoamine) (PAMAM) dendrimers in solution," Langmuir, vol. 25, pp. 3271-3275, 2009

[7] D. Lombardo, P. Calandra, S. Magazù, et al. "Soft nanoparticles charge expression within lipid membranes: the case of amino terminated dendrimers in bilayers vesicles". Colloid Surface B, vol. 170, pp. 609-616, 2028.

[8] D. Lombardo, P. Calandra, M.T. Caccamo, at al. "Colloidal stability of liposomes," AIMS Mater. Sci. vol. 6, pp. 200. 2018

[9] N. Micali, L.M. Scolaro, A. Romeo, D- Lombardo, P. Lesieur, F. Mallamace, "Structural properties of methanolpolyamidoamine dendrimer solutions," Phys. Rev. E, vol. 58, pp. 6229-6235

[10] D. Lombardo, P. Calandra, E. Bellocco et al., "Effect of anionic and cationic polyamidoamine (PAMAM) dendrimers on a model lipid membrane," Biochim Biophys Acta Biomembr, vol. 1858, pp. 2769-2777. 2016.

[11] D. Astruc, F. Chardac, "Dendritic Catalysts and Dendrimers in Catalysis," Chem Rev, vol. 101, pp. 2991-3024, 2001.

[12] D. Astruc, B. Boisselier, C. Ornelas, "Dendrimers designed for functions: from physical, photophysical, and supramolecular properties to applications in sensing, catalysis, molecular electronics, photonics, and nanomedicine", Chem. Rev., vol. 110, pp. 1857-1959, 2010.

[13] M. Nemanashi, J. Noh, R. Meijboom, "Dendrimers as alternative templates and pore-directing agents for the synthesis of micro- and mesoporous materials," J. Mater. Sci., vol. 53, pp. 12663-12678, 2018.

[14] L. Bonaccorsi, D. Lombardo, A. Longo, E. Proverbio, A. Triolo, "Dendrimer template directed self-assembly during zeolite formation," Macromolecules, vol. 42, pp. 1239-1243, 2009.

[15] L. Bonaccorsi, P. Calandra, H. Amenitsch, E. Proverbio, D. Lombardo, Growth of fractal aggregates during template directed SAPO-34 zeolite formation. Micropor. Mesopor. Mat. Vol. 167, pp. 3-9, 2013.

[16] R. M. Kannan1, E. Nance2, S. Kannan2 \& D. A. Tomalia, Emerging concepts in dendrimer-based nanomedicine: from design principles to clinical applications, Journal of Internal Medicine, , 276; 579-617, 2014.

[17] H.B Harris, B.L. Turner, Dendrimers: Synthesis, Applications and Role in Nanotechnology, Nova Science Pub Inc, (2013)., 